\title{
Reliable evidence for efficacy of single dose oral analgesics
}

\section{Abstracted from}

Moore RA, Derry S, Aldington D, Wiffen PJ.

Single dose oral analgesics for acute postoperative pain in adults - an overview of Cochrane reviews. Cochrane Database Syst Rev 2015; 9: CD008659. DOI: 10.1002/14651858.CD008659.pub3.

Address for correspondence: Sheena Derry, Pain Research and Nuffield Department of

Clinical Neurosciences (Nuffield Division of Anaesthetics), University of Oxford,

Pain Research Unit, Churchill Hospital, Oxford, Oxfordshire, OX3 7LE, UK. E-mail: sheena.derry@ndcn.ox.ac.uk.

\section{Question: What is the efficacy of single dose oral analgesics in adults with at least moderate pain following surgery?}

Data sources The Cochrane library was searched for Cochrane systematic reviews.

Study selection Cochrane reviews on single pain medications for the treatment of acute pain were included. Non-Cochrane reviews were included for tramadol.

Data extraction and synthesis Two reviewers independently searched, selected reviews for inclusion, assessed quality and performed data extraction. A protocol in case of disagreement was in place. Data were collected on number of included studies and participants, drug, dose and formulation and pain model. The authors concentrated on the amount of information and the potential for publication bias.

Pain relief was calculated using at least 50\% maximum pain relief, as a percentage, and as NNTs. Duration of analgesia was measured as mean or median and time to remedication was calculated as percentage of patients.

Results Thirty-nine reviews including 41 interventions were analysed and NNTs for at least $50 \%$ maximum pain relief were summarised in a graphic. NNTs range from almost one all the way to five. Only one intervention, codeine 60 , had an NNT $\geq 10$. Results judged to be reliable were listed in detail. Mean or median time to remedication was also presented in a graphic.

The authors conclude that there is a great amount of quality information on single dose analgesics, and highlighted the potential benefit of fast acting formulations and fixed formulations to achieve good long-lasting analgesia.

Conclusions There is a wealth of reliable evidence on the analgesic efficacy of single dose oral analgesics. Fast acting formulations and fixed dose combinations of analgesics can produce good and often long-lasting analgesia at relatively low doses. There is also important information on drugs for which there are no data, inadequate data, or where results are unreliable due to susceptibility to publication bias. This should inform choices by professionals and consumers.

This paper is based on a Cochrane Review published in the Cochrane Library 2015, issue 9 (see www.thecochranelibrary.com for information). Cochrane Reviews are regularly updated as new evidence emerges and in response to feedback, and the Cochrane Library should be consulted for the most recent version of the review.

\section{Commentary}

This high quality review is the update from a previous version on the topic from 2011, addressing the efficacy of the use of single dose oral analgesics on the treatment of moderate to severe pain.

The authors searched the Cochrane library for Cochrane reviews on the use of postoperative oral pain medications and identified new reviews and some updates.

Two authors independently searched, reviewed for inclusion, assessed quality and extracted data. There was a protocol in place in case of disagreement.

They collected information on number of studies and participants, drug dose and formulation and pain model.

The reviewers counted on the fact that each Cochrane review follows a precise standard quality protocol, so the authors concentrate on publication bias and, together with the amount of information help establish a quality classification of the evidence.

Unlike in the previous version, dental and non-dental studies were combined.

The authors included 39 Cochrane reviews of 41 single analgesics or combinations. They explained that the oldest reviews (1999 and 2000) were updated in 2008 with no new information. They also highlighted the need for an update on ibuprofen and they recognise the lack of a Cochrane review on the popular pain medication tramadol.

Impressively, 467 individual studies are included in this review with an approximate 50,000 patients. The authors carefully reviewed the conclusions in each individual review. These reviews only included high quality studies of standardised designs and methods.

The authors collected available risk ratios with confidence intervals and NNTs or calculated 50\% pain relief over four to six hours. Time to remedication was also calculated. No NNTs were calculated if the CI included 1 on the RR.

They set a cut-off point for NNT at $\geq 10$ for clinical relevance for studies showing no effect as a means to assess likelihood of publication bias. The authors chose this approach claiming there is no helpful statistical test to show publication bias.

Results were presented as NNTs for at least 50\% maximum pain relief. From the top ten with lowest NNTs two are COX2 inhibitors (etoricoxib), three include ibuprofen in combination with paracetamol (400/1000 or 200/500) and caffeine (200/100), two have diclofenac $\mathrm{K}$ formulations (100 and 50), one is for ketoprofen (25), one for diflunisal (1000). There is only one opioid in combination that is part of the top ten: paracetamol +oxycodone (1000/10). 
Other important results are provided as time to remedication. From the top ten interventions with the longest time to remediation four are COX2 inhibitors, two are diflunisal formulations (1000 and 500), two are paracetamol and oxycodone (650/10 and $1000 / 10)$, one is for naproxen $(500 / 550)$ and one for ibuprofen in combination with paracetamol (400/1000). Percentage of remedication was also calculated and presented in graphics without naming the individual agents.

Despite this impressive amount of data there are several unanswered issues since adverse events, interactions, preferences and costs are not part of this review. Information on adverse effects are however available from a companion review. ${ }^{1}$ In the real world it is very unlikely that a patient will require only a single dose of a pain medication.

Silvia Spivakovsky and Yael Spivakovsky New York University College of Dentistry, New York, USA

1. Moore RA, Derry S, Aldington D, Wiffen PJ. Adverse events associated with single dose oral analgesics for acute postoperative pain in adults - an overview of Cochrane reviews. Cochrane Database Syst Rev 2015; 10: CD011407. DOI: 10.1002/14651858. CD011407.pub2.

Evidence-Based Dentistry (2016) 17, 60-61. doi:10.1038/sj.ebd.6401175 